\title{
Polymerase theta-helicase promotes end joining by stripping single-stranded DNA-binding proteins and bridging DNA ends
}

\author{
Jeffrey M. Schaub ${ }^{1, *}$, Michael M. Soniat ${ }^{1}$, and Ilya J. Finkelstein ${ }^{1,2, *}$ \\ ${ }^{1}$ Department of Molecular Biosciences and Institute for Cellular and Molecular Biology, The University of Texas at Austin, \\ Austin, Texas 78712, USA \\ ${ }^{2}$ Center for Systems and Synthetic Biology, The University of Texas at Austin, Austin, Texas 78712, USA \\ * To whom correspondence should be addressed: \\ Jeffrey M. Schaub: jmschaub@gmail.com \\ Ilya J. Finkelstein: ilya@ finkelsteinlab.org
}

\begin{abstract}
Homologous recombination-deficient cancers rely on DNA polymerase Theta (Pol $\theta$ )-Mediated End Joining (TMEJ), an alternative double-strand break repair pathway. Pol $\theta$ is the only vertebrate polymerase that encodes an N-terminal superfamily 2 (SF2) helicase domain, but the role of this helicase domain in TMEJ remains unclear. Using single-molecule imaging, we demonstrate that Pol $\theta$ helicase (Pol $\theta-h$ ) is a highly processive single-stranded DNA (ssDNA) motor protein that can efficiently strip Replication Protein

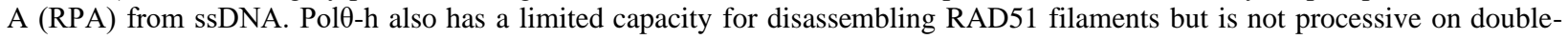

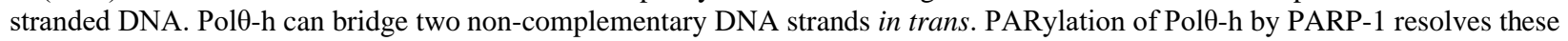

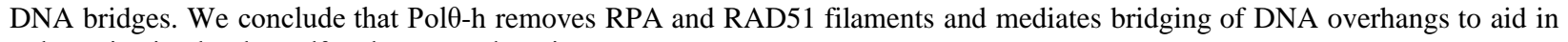
polymerization by the Pol $\theta$ polymerase domain.
\end{abstract}

Keywords: SF2 helicase, Replication Protein A, RAD51, PARP-1, PARylation, single-molecule, DNA curtains

\section{Introduction}

DNA double-strand breaks (DSBs) are highly toxic lesions that occur during cellular metabolism and in response to cancer therapies. Non-homologous end-joining (NHEJ) - the predominant DSB repair pathway in human cells - initiates when the ring-like Ku70/80 heterodimer binds the free DNA ends $(1,2)$. Subsequently, Ku recruits additional repair factors to the DSB, including DNA-PKcs to bridge the DNA ends and ligases to seal the break (3, 4). Homologous recombination (HR) is an error-free repair pathway that partially processes the free DNA ends to expose 3' -single-stranded DNA (ssDNA) overhangs (5). These overhangs are rapidly bound by replication protein A (RPA). Subsequently, RAD51 replaces RPA on the ssDNA to search for sequence homologies in a sister chromatid (6). RAD51-mediated strand invasion facilitates templated polymerization of a homologous DNA sequence (7). While NHEJ is active throughout the cell cycle, HR is restricted to the $S$ and $G 2$ phases of the cell cycle when a homologous template is available $(8,9)$.

Many cancer types accumulate mutations in NHEJ- or HRdependent proteins and become reliant on theta-mediated end-joining (TMEJ), an error-prone DSB repair pathway (10). TMEJ is mediated by DNA Polymerase Theta (Pol $\theta)$, PARP-1, and DNA Ligase III, as well as traditional DNA resection factors (11-13). Unlike HR, TMEJ requires short microhomologies (2-6 bp) and is highly mutagenetic, leading to increased chromosomal rearrangement and short insertions/deletions (14-16). During TMEJ, 3'-ssDNA overhangs are generated by MRE11RAD50-NBS1 complex (MRN)/CtIP-mediated resection and are annealed at microhomologies (17). The resulting flaps are nucleolytically removed, and Pol $\theta$ further extends the junctions to stabilize the microhomology (18). However, resected ssDNA is rapidly bound by
RPA, which blocks the annealing of microhomologies (19). Furthermore, the recombinase RAD51 displaces RPA with the help of BRCA2 and other recombination mediators (20). Inactivation of TMEJ leads to an increase in HR, suggesting that these two repair pathways are antagonistic $(21,22)$

$\operatorname{Pol} \theta$ is evolutionarily conserved across higher eukaryotes but is missing in fungi (23). Pol $\theta$ encodes an $\mathrm{N}$-terminal superfamily 2 (SF2) helicase/ATPase domain, a central disordered domain, and a C-terminal A-family polymerase domain (Figure 1A) $(24,25)$. The isolated Pol $\theta$ -

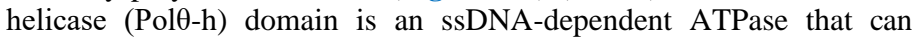
unwind short DNA duplexes and displace RPA from oligo-length DNA substrates in vitro $(26,27)$. In cells, Pol $\theta$-h mutants increase the prevalence of RAD51 foci after radiation exposure and shift the spectrum of end-joining products with microhomologies near the 3' ends of DNA substrates $(15,21)$. This polymerase is frequently overexpressed in cancers deficient in traditional DSB repair mechanisms, and elevated

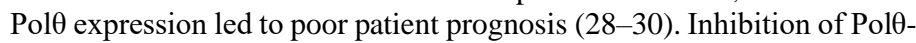
$\mathrm{h}$ can kill HR-deficient tumor cells, suggesting a therapeutic route for targeting such malignancies (31). Pol $\theta$ is an especially promising therapeutic target when combined with PARP-1 inhibitors in NHEJ/HRdeficient cancers $(21,22,31-33)$. Together, these studies have

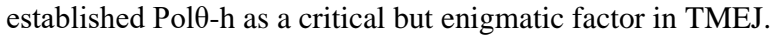

Here, we use single-molecule and ensemble biochemical

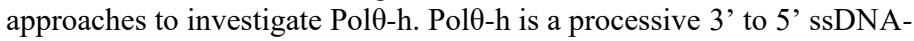
binding motor and can readily displace RPA from ssDNA. Pol $\theta$-h can also partially disassemble RAD51 filaments, although this activity is much lower than its ability to remove RPA. Additionally, Pol $\theta$-h can bridge two DNA molecules that mimic resection intermediates in trans in a reaction that does not require ATP, suggesting that the 
homotetrameric assembly may tether two arms of a double-strand break during TMEJ. These DNA bridges were resistant to high salt, suggesting additional protein factors may be required for DNA dissociation. Therefore, we investigated the role of PARP-1 regulation of Pol $\theta$. PARP1 rapidly binds to DNA damage sites and initiates the synthesis of poly

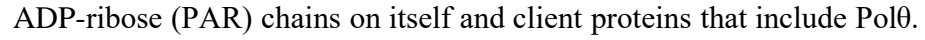
We show that PARP-1 PARylates Poly-h in vitro and reduces the ssDNA binding affinity and promotes dissociation. We conclude that PARP-1 may regulate Pol $\theta$-h activity to promote DNA polymerization after the microhomology is established.
A

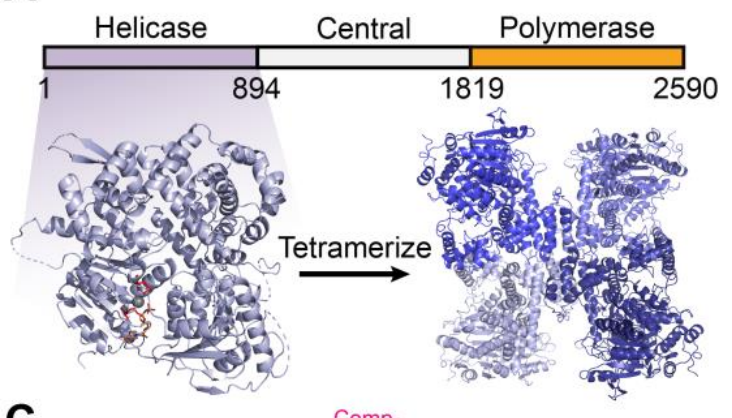

C

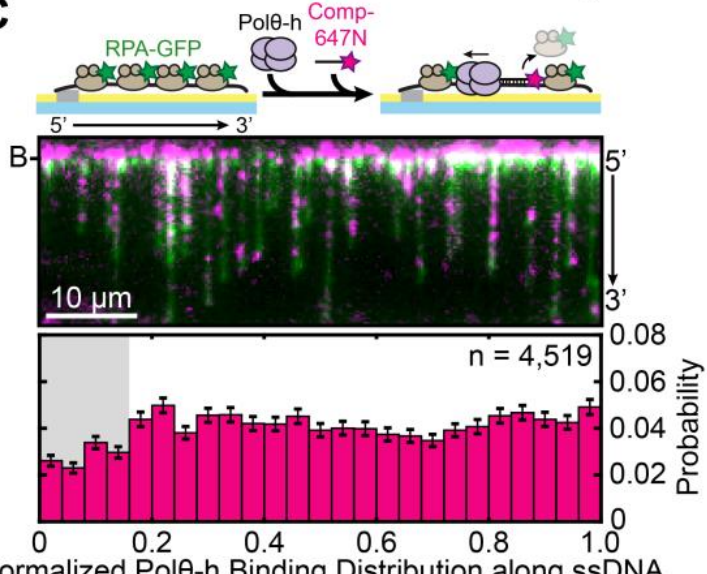

Normalized Pole-h Binding Distribution along ssDNA

D
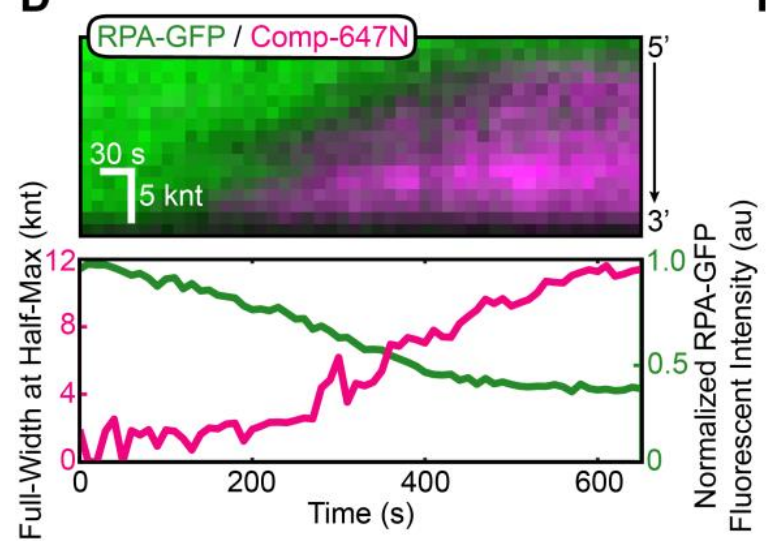

B

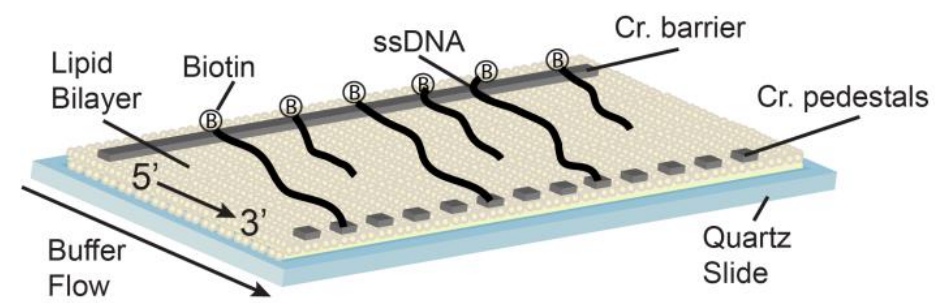

E

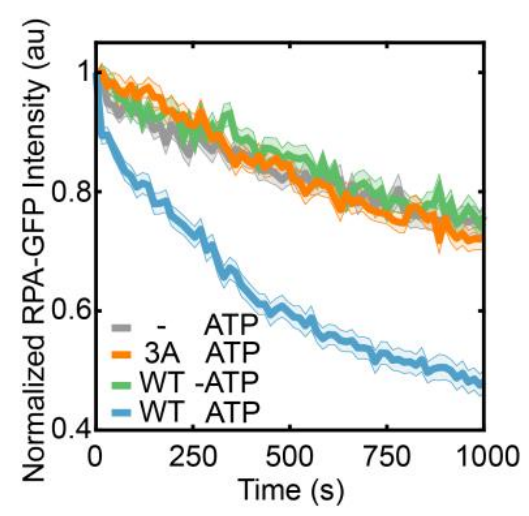

$\mathbf{F}$

G
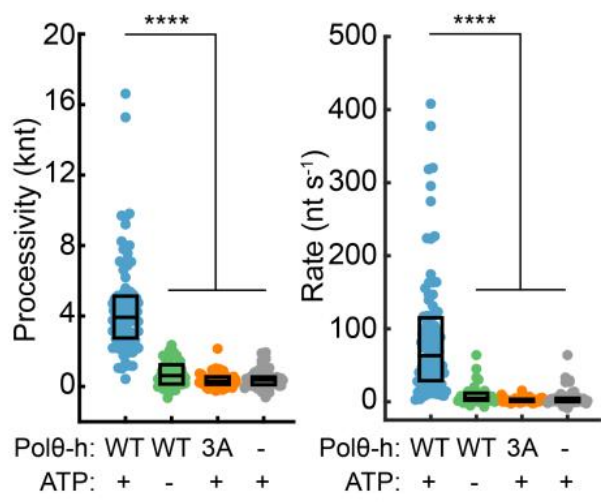

H

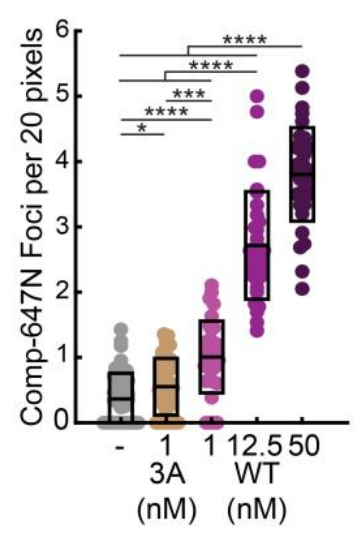

I

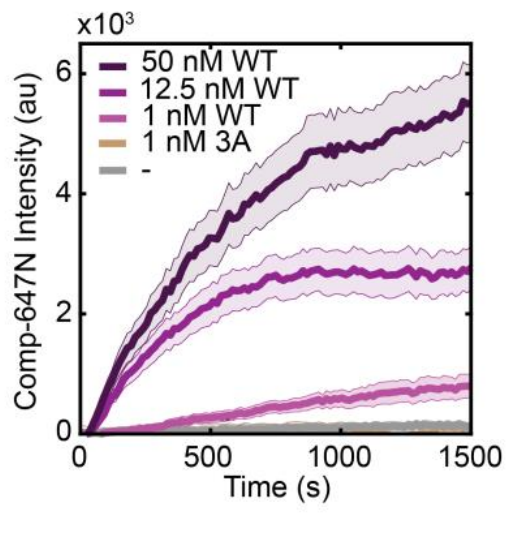

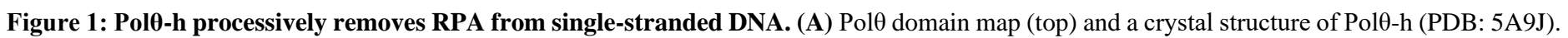
(B) Schematic of ssDNA curtains assay. For double-tethered ssDNA curtains, buffer flow is stopped after DNA molecules are immobilized between

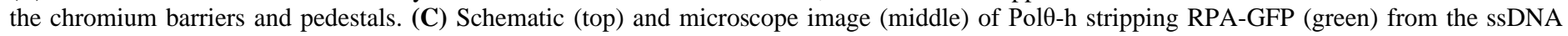

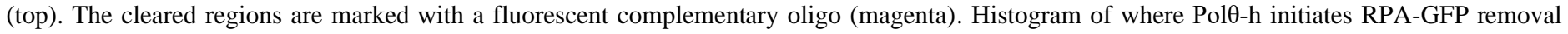
(bottom). (D) (top) Kymograph of processive RPA-GFP (green) removal, as measured by fluorescent complementary oligonucleotide (magenta). Bottom: Analysis of Pol $\theta$-h translocation (magenta) and RPA-GFP fluorescence intensity (green). (E) Normalized RPA-GFP fluorescent intensity for

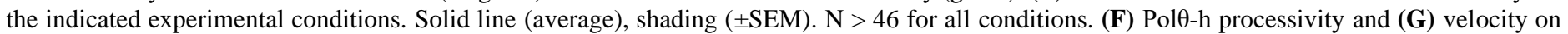
RPA-coated ssDNA. Box displays median and IQR. N > 46 for all conditions. (H) Quantification of fluorescent oligonucleotide foci for each Pol $\theta-h$

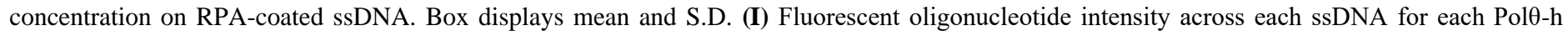
concentration. Solid line (average), shading ( \pm SEM). 


\section{Results}

\section{Polt-helicase strips RPA from single-stranded DNA.}

We purified and confirmed that the Pol $\theta$ helicase domain (amino acids 1-894, referred to as Poly-h) assembles into homotetramers via calibrated size exclusion chromatography consistent with previous studies (Figure 1A, Figure S1) (34). Next, we monitored single Poly-h complexes using single-stranded DNA (ssDNA) curtains (Figure 1B) $(35,36)$. In this assay, ssDNA is generated by rolling-circle amplification of a repeating 28-nucleotide minicircle with low structural complexity $(37,38)$. The 5 ' end of the primer includes biotin and the resulting ssDNA molecule is immobilized on the surface of a fluid lipid bilayer via biotinstreptavidin interactions. The ssDNA is then extended from the tether point via mild buffer flow.

We first assayed how Pol $\theta$-h counteracts RPA-coated ssDNA because RPA inhibits hybridization of heteroduplex oligos during TMEJ (19). We monitored the removal of fluorescent RPA-GFP because multiple fluorescent labeling strategies resulted in hypoactive Pol $\theta-h$ (Figure S2A) (39). In this assay, ssDNA curtains are assembled with RPA-GFP. Next, unlabeled Poly-h is added to the flowcell, and unbound protein is washed out. RPA clearance is observed following injection of fluorescent complementary oligonucleotide that can tile across the ssDNA substrate (Comp-647N) (Figure 1C). Injecting PolO-h into the flowcell created a punctate pattern with reduced RPA-GFP signal and increased fluorescent oligonucleotide binding. RPA clearance and oligo binding required Pol $\theta-\mathrm{h}$, suggesting that the helicase clears the ssDNA by removing RPA. Pol $\theta$-h cleared RPA along the entire ssDNA molecule, with a slight decrease at the 5' end due to optical interference from the chromium barrier (Figure 1C, Figure S2D).

Next, we quantified RPA removal on double-tethered ssDNA curtains. RPA-ssDNA is tethered to downstream microfabricated chromium features and buffer flow is then stopped to observe protein dynamics on the double-tethered ssDNA in the absence of hydrodynamic

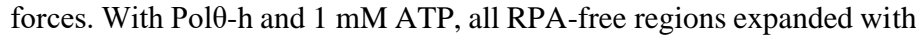
a 3' to 5' polarity ( $\mathrm{N}=91 \mathrm{Pol} \theta-\mathrm{h}$ molecules), consistent with other SF2family helicases (27, 40) (Figure 1D). This RPA-cleared zone was rapidly hybridized by Comp-647N, indicating that Pol $\theta$-h created RPAfree ssDNA regions. RPA-GFP intensity decreased more rapidly in the

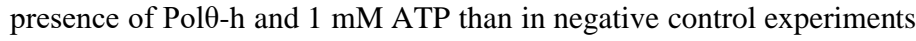
without ATP or an ATPase-dead Pol0-h (E121A, D216A, and E217A; termed the 3A mutant) (21) (Figure 1E). We also observed small Comp$647 \mathrm{~N}$ puncta when Pol0-h and/or ATP were omitted from the reaction. These foci were static throughout the experiment and likely represent locations where RPA-GFP is transiently displaced by excess Comp-647N (Figure S2E, Table S1). To estimate the processivity and rate of Pol $\theta-h$ translocation, we fit the Comp-647N signal to a Gaussian function and calculated the full-width at half-max for each time point (Figure S2B). $\mathrm{Pol} \theta-\mathrm{h}$ is a processive enzyme, clearing $\sim 3.9$ kilonucleotides $(\mathrm{knt}$; IQR = 2.7-5.1 knt; N=91 Pol $\theta-\mathrm{h}$ molecules) of RPA-coated ssDNA with a median velocity of $63 \mathrm{nt} \mathrm{s}^{-1}\left(\mathrm{IQR}=28-117 \mathrm{nt} \mathrm{s}^{-1}, \mathrm{~N}=91\right)$ (Figure 1F,G). Increasing Pol $\theta-h$ concentration increased the number of Comp-647N
A
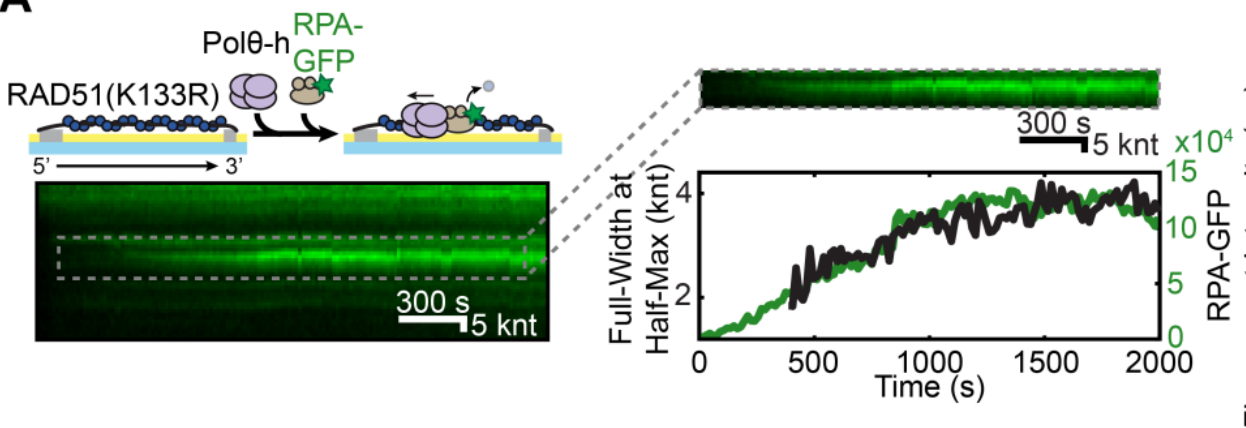

C

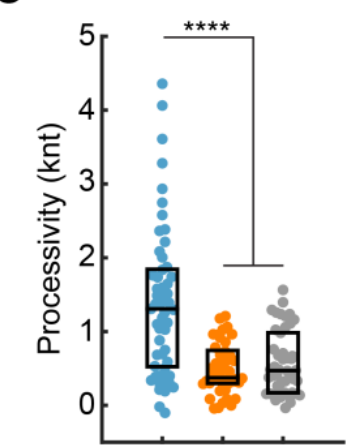

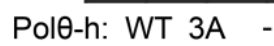

D

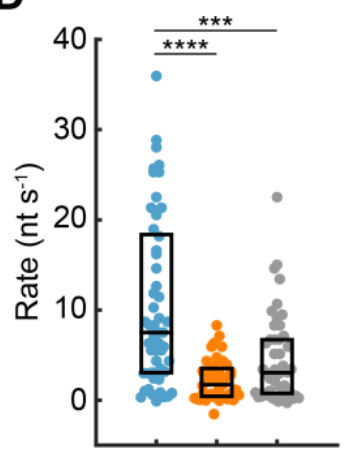

PolO-h: WT 3A -
E

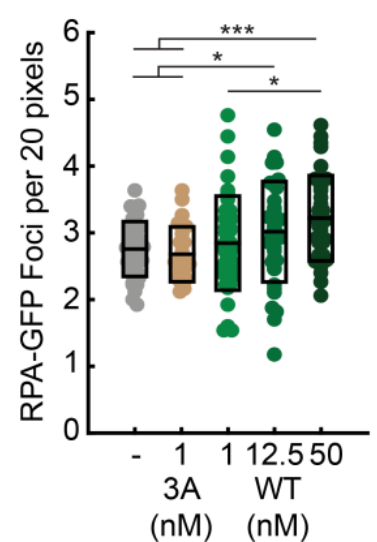

B

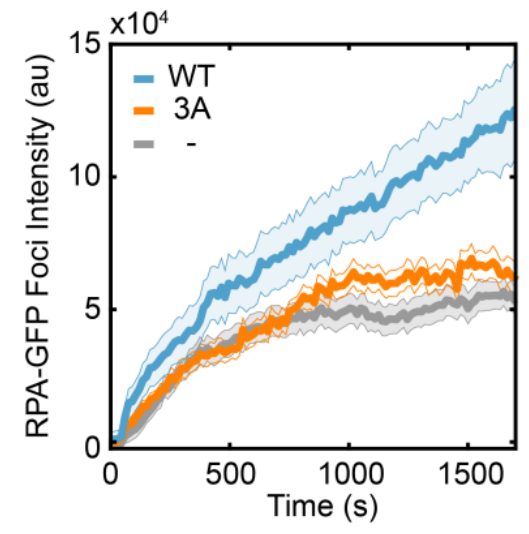

$\mathbf{F}$

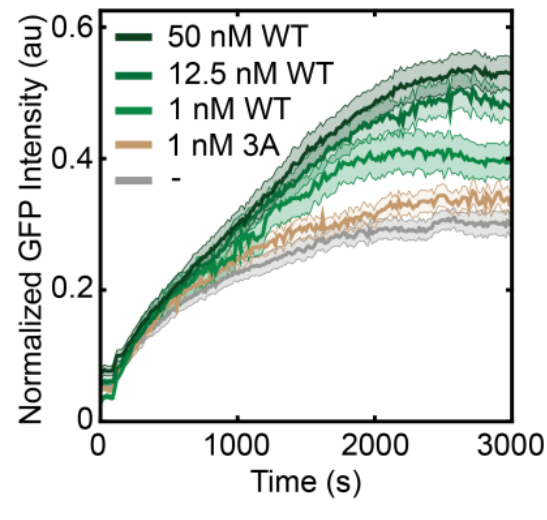

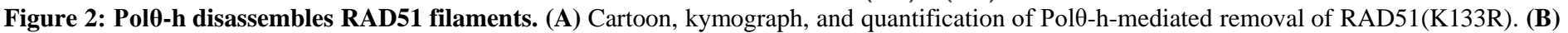
Quantification of the RPA-GFP foci fluorescent intensity over time. Solid line (average), shading ( \pm SEM). N $>36$ for all conditions. (C) Pol $\theta-h$ processivity and (D) velocity on RAD51(K133R)-coated ssDNA. Box displays median and IQR. N> 36 for all conditions. (E) Quantification of RPA-

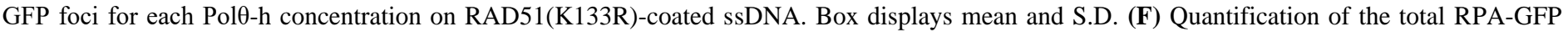

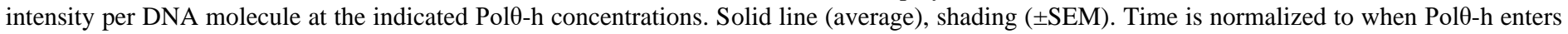
the flowcell ( $\mathrm{t}=0) .2 \mathrm{nM}$ RPA-GFP is immediately injected after, and flow is stopped at $\mathrm{t}=100 \mathrm{~s}$. Fluorescent intensity along ssDNA molecules is normalized to the initial RPA-GFP intensity prior to RAD51(K133R) displacement. 
Schaub et al. 2021 - preprint copy - BioRxiv

foci per unit length and increased the total Comp-647N fluorescence intensity along the ssDNA substrate (Figure 1H, I, Figure S2F, Table

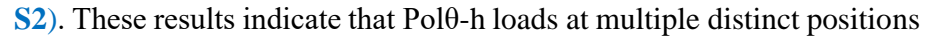
along the ssDNA substrate. Taken together, we show that Pol $\theta-h$ is a processive 3' to 5' ssDNA motor that uses ATP hydrolysis to strip RPA from ssDNA.

Poly-h can unwind short duplex DNA molecules and DNARNA hybrids with limited processivity (27). Having observed processive ssDNA translocation, we next tested whether Poly-h is also a processive helicase. We used a double-stranded DNA (dsDNA) substrate with 3'ssDNA overhangs that mimic TMEJ resection intermediates. Helicase activity generates ssDNA that can be monitored via a growing RPA-GFP signal (Figure S3A) (41). However, the RPA-GFP intensity did not

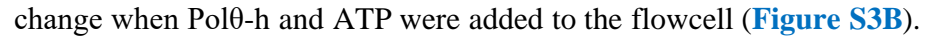
Although we cannot rule out limited helicase activity below our $\sim 500$ bp resolution, we conclude that $\mathrm{Pol} \theta-\mathrm{h}$ is not a processive helicase on dsDNA $(25,27,34)$.

\section{Polt-h poorly disassembles RAD51 filaments}

In addition to clearing RPA, Pol $\theta$ has been proposed to antagonize HR by removing RAD51 filaments from $\operatorname{ssDNA}(21,22)$. To test this hypothesis, we developed an assay to monitor Pol $\theta$-h-dependent RAD51 removal. RAD51 turnover on ssDNA is stimulated by its intrinsic ATPase activity but can be inhibited by adding $\mathrm{Ca}^{2+}$ to stabilize

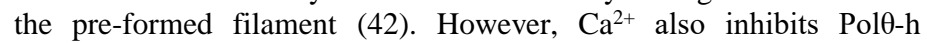
translocation on ssDNA (Figure S4A). Therefore, we used the ATPasedeficient RAD51(K133R) to stabilize RAD51 on ssDNA with ATP and $\mathrm{Mg}^{2+}$ in the reaction buffer (Figure S4B, C) (43). This mutation disrupts the Walker B ATPase motif, permitting ATP binding but not hydrolysis. We confirmed that RAD51(K133R) rapidly displaces RPA-GFP from ssDNA similarly to wild-type RAD51, albeit with a slightly longer nucleation phase (Figure S4D). As expected, RAD51(K133R) filaments are also more stable than WT RAD51 when challenged with RPA-GFP in the presence of $\mathrm{Mg}^{2+}$ and ATP (Figure S4E). In sum, RAD51(K133R) filaments assemble on ssDNA but remain stable in a buffer that also supports Pol $\theta$-h translocation.

We next tested whether Pol $\theta$-h can strip pre-formed RAD51(K133R) filaments from ssDNA. We first coated the ssDNA with RAD51(K133R) and then injected Pol $\theta$-h with a low concentration of RPA-GFP to visualize any ssDNA that is created during RAD51 removal (Figure 2A). In the presence of Pol $\theta$-h, the RPA-GFP puncta were $\sim 2-$ fold brighter $(\mathrm{N}=53)$ than $\operatorname{Pol} \theta-\mathrm{h}(3 \mathrm{~A})$ and when $\mathrm{Pol} \theta-\mathrm{h}$ was omitted ( $\mathrm{N}=41$ and $\mathrm{N}=46$, respectively) (Figure 2B). On RAD51(K133R)-coated ssDNA, the median Pol $\theta-\mathrm{h}$ processivity was $1.3 \mathrm{knt}(\mathrm{IQR}=0.5-1.9 \mathrm{knt}$, $\mathrm{N}=53)$ and the velocity was $8 \mathrm{nt} \mathrm{s}^{-1}\left(\mathrm{IQR}=3-19 \mathrm{nt} \mathrm{s}^{-1}, \mathrm{~N}=53\right)$ (Figure 2C, D, Table S3). Processivity was reduced 3 -fold and the velocity was 8-fold slower with RAD51(K133R) as compared to RPA. In contrast to

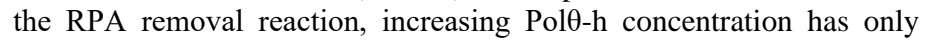
modest effects on the number of RPA-GFP foci per ssDNA (Figure 2E, Figure S4F, Table S4). The total RPA-GFP fluorescent intensity along the entire ssDNA substrate increased only $\sim 2$-fold above control experiments with inactive Pol $\theta$-h (Figure 2F). These results indicate that

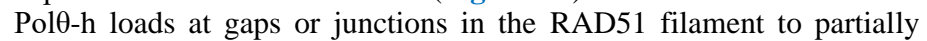
disassemble stabilized RAD51 filaments.

\section{PARP-1 reverses Polt-h-mediated DNA bridges}

TMEJ initiates after broken DNA ends are resected to reveal ssDNA overhangs (17). Pol $\theta$ is proposed to bridge these overhangs
A
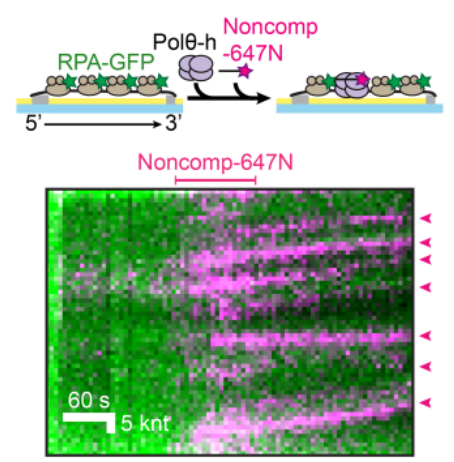

C

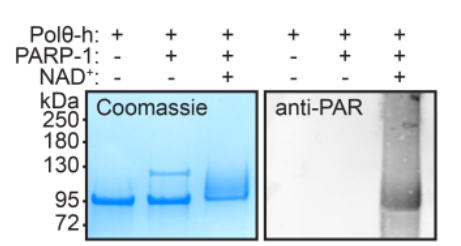

D

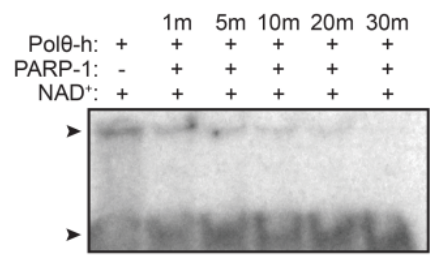

E

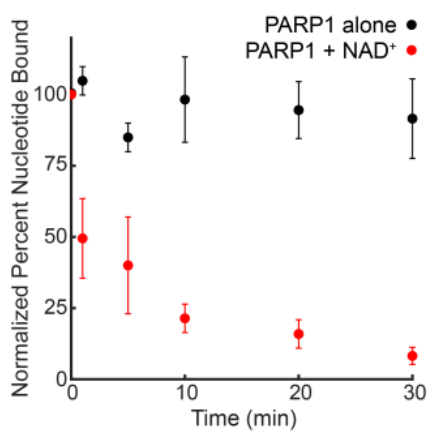

B

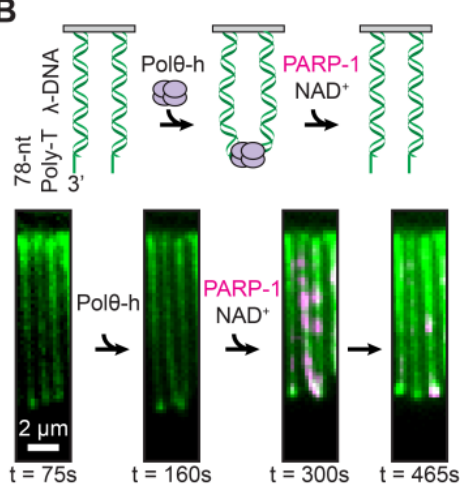

$\mathbf{F}$

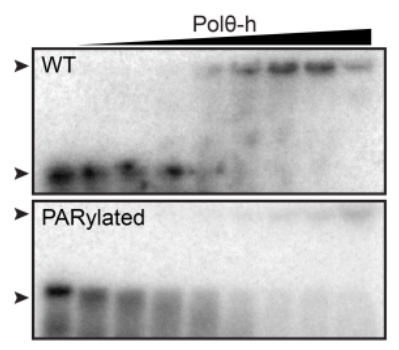

G

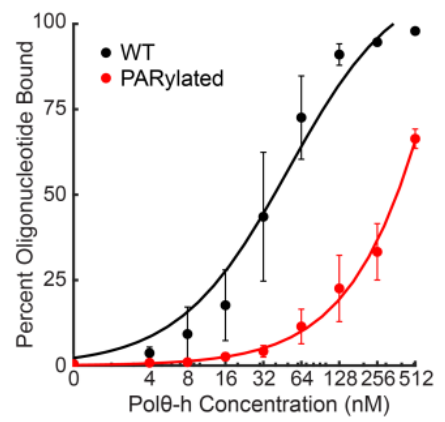

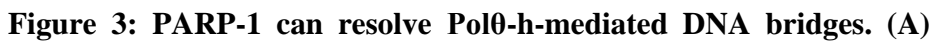
Cartoon (top) and kymographs (bottom) of Poll-h-mediated tethering of two non-complementary ssDNA molecules. The long ssDNA is labeled with RPA-GFP (green) and the short non-complementary oligo is labeled with Atto647N (magenta). (B) PolO-h tethers DNA molecules with 3'ssDNA overhangs that mimic resected ends. Cartoon of DNA end bridging (top) and images of two tethered $\lambda$-phage DNA molecules. DNA is visualized with YOYO-1 (green). Addition of PARP-1 (magenta) and $\mathrm{NAD}^{+}$dissociates these bridges. (C) Coomassie (left) and a western blot

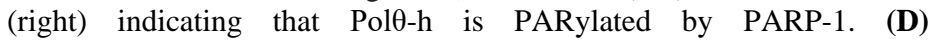

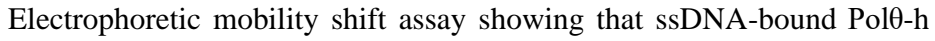

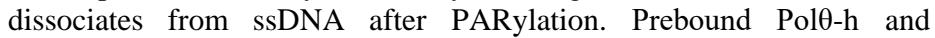
radiolabeled ssDNA oligonucleotide were incubated with PARP-1 and $\mathrm{NAD}^{+}$for the indicated times. Arrows indicate unbound and bound oligonucleotide. (E) Quantification of (D). Binding normalized to condition without PARP-1. Average of three replicates. Error $=$ SEM. $(\mathbf{F})$ WT and PARylated Poly-h EMSA on a radiolabeled ssDNA oligonucleotide. Pol $\theta$-h concentrations range from 0 to $512 \mathrm{nM}$. Arrows indicate unbound and bound oligonucleotide. (G) Quantification of (F). Fit to hyperbolic functions. Average of three replicates. Error $=\mathrm{SEM}$. 
despite their limited homology. The homotetrameric assembly of the helicase domain may underpin this multivalent DNA binding (34). To

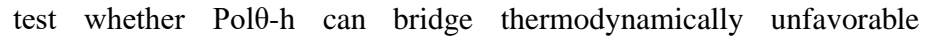

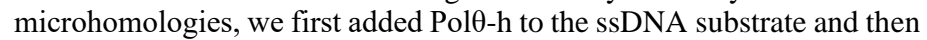
flowed in a fluorescent non-complementary oligonucleotide (Noncomp647N) (Figure 3A). Pol $\theta$-h efficiently captured this oligo, indicating that

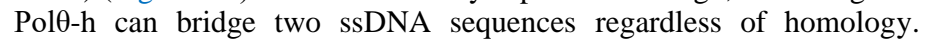
Notably, oligo capture required Pol $\theta-h$, whereas oligos did not associate

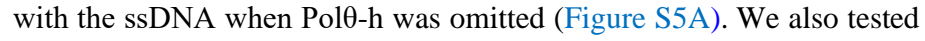

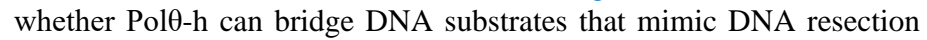
intermediates. We assembled $48 \mathrm{kbp}$-long dsDNAs with a 3'-T78 ssDNA overhang. Adding $5 \mathrm{nM}$ Pol $\theta$-h resulted in bridging of adjacent molecules at their free DNA ends (Figure S5B). DNA bridging required Pol $\theta-h$ but

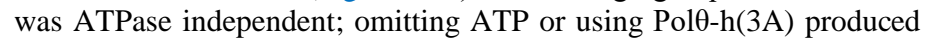
indistinguishable end-tethered DNAs (Figure S5C). These bridges persisted for the duration of the 15-minute imaging experiment. We additionally injected a $\sim 60 \mathrm{~s}$ pulse of $1 \mathrm{M} \mathrm{NaCl}$ to attempt to dissociate

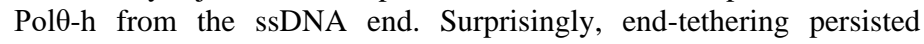
through the high salt wash (Figure S5B).

We reasoned that Pol $\theta$-h-DNA bridges must be resolved for downstream TMEJ by another protein factor. PARP-1 is an attractive candidate for this activity for three reasons. First, PARP-1 is one of the earliest enzymes to arrive at broken DNA ends and plays a critical role in promoting TMEJ $(13,44,45)$. Second, poly-ADP-ribosylation of client proteins by PARP-1 results in their release from DNA (46-49). Third, a proteomics screen identified the N-terminus of Pol $\theta$ (i.e., the helicase domain) as a PARylation target (50). Consistent with our hypothesis, adding PARP-1 and NAD ${ }^{+}$dissolved resected DNA bridges (Figure 3B). Omitting either PARP-1 or NAD ${ }^{+}$was not sufficient to resolve these DNA bridges alone (Figure S5B). Purified PARP-1 can also PARylate

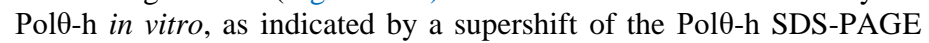
band upon incubation with PARP-1 and $\mathrm{NAD}^{+}$(Figure 3C, Figure S5D, E) An anti-PAR western blot confirmed that the upshifted Pol $\theta$-h band represents a PARylated product.

We further quantify whether PARylated Pol $\theta$-h has impaired ssDNA binding relative to the unmodified enzyme using electrophoretic mobility shift assays (EMSAs). Pol $\theta-h$ was pre-incubated with a radiolabeled $\mathrm{dT}_{50}$ oligonucleotide prior to the addition of PARP-1 and $\mathrm{NAD}^{+}$. ssDNA-bound Pol $\theta-\mathrm{h}$ is rapidly released from ssDNA upon PARylation (Figure 3D, E). ssDNA remained bound by Pole-h in the presence of only PARP-1 (Figure 3E, Figure S5F). We also changed the order of addition by pre-incubating Pol $\theta-h$ with PARP- 1 and NAD ${ }^{+}$prior to incubating with a radiolabeled $\mathrm{dT}_{50}$ oligonucleotide (Figures $3 \mathrm{~F}$ ).

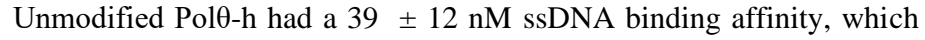
closely matches the $30 \mathrm{nM}$ affinity measured via fluorescence anisotropy

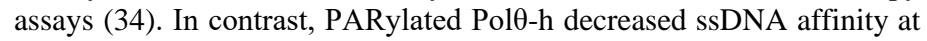
least ten-fold compared to Pol $\theta$-h alone (> $370 \mathrm{nM}$ ) (Figure 3G). Taken together, the single-molecule and ensemble experiments demonstrate that PARP-1 can PARylate Pol $\theta-h$ and that PARylation reduces the ssDNA binding affinity of Pol $\theta-h$.

\section{Discussion}

Figure 4 summarizes our model for how Pol $\theta$ uses its unique helicase domain during TMEJ. Pol $\theta$ encounters RPA-coated ssDNA that is generated during resection. Its helicase domain translocates in a 3' to 5' direction to processively remove RPA and other ssDNA-binding proteins from the ssDNA substrate. RPA prevents the hybridization of

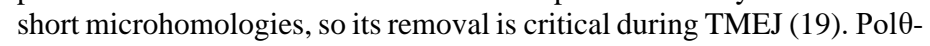
$h$ removes RPA over thousands of nucleotides and can also partially disassemble RAD51 filaments. We used a RAD51 mutant that stabilizes ssDNA filaments in these studies, so these results are likely a lower

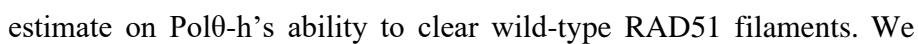
conclude that the helicase domain can load within RPA-coated segments or at RAD51-RPA filament junctions to rapidly remove RAD51 over the tens to hundreds of nucleotides that are required to synapse TMEJ junctions in cells (51-53). After clearing the ssDNA, Pol $\theta-h$ bridges two DNA ends. This may be sufficient for the polymerase domain to extend the microhomologies. Following polymerization, these bridges can be resolved by PARP-1-dependent Pol $\theta$ PARylation, which reduces the affinity of the enzyme for ssDNA. Removing Pol $\theta$ may be required for ligases to re-seal the broken DNA breaks.

By removing RPA, Pol $\theta$ accomplishes the twin goals of exposing short microhomologies and suppressing the RPA to RAD51

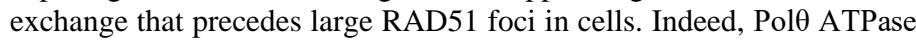
mutants that disrupt the helicase domain shift the spectrum of TMEJ junctions to external microhomologies in cells. This result is presumably due to its inability to processively translocate and remove ssDNA-
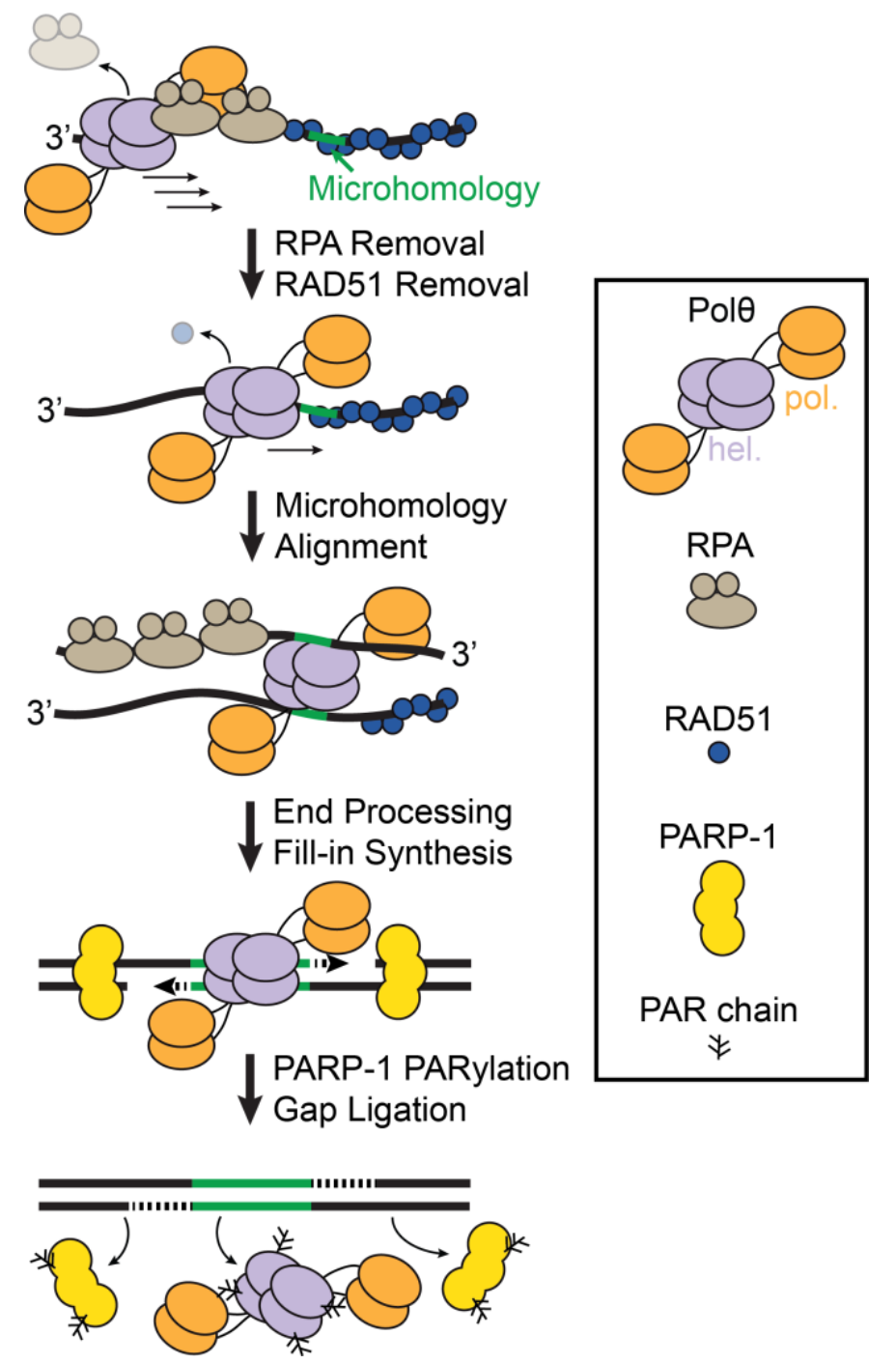

Figure 4: Model of PolO activities in Theta-mediated end-joining.

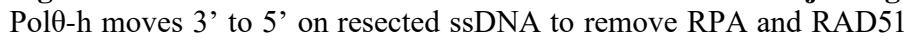
and scans for microhomologies (green). 3' ends are processed and resulting gaps are filled in by the Pol $\theta$ polymerase domain. PARP-1 PARylates Pol $\theta$ to remove it from DNA after gap filling. 
Schaub et al. 2021 - preprint copy - BioRxiv

binding proteins to expose internal microhomologies $(15,51)$. We also observed that Polt-h loads more efficiently on RPA- vs RAD51-coated ssDNA in a concentration-dependent manner. RPA rapidly exchanges and diffuse on ssDNA and we propose that this dynamic nature may

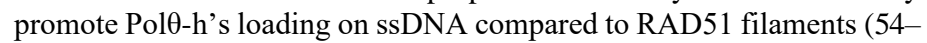
56). Our observation that Pol $\theta$-h has limited RAD51 clearing activity is consistent with previous studies, including reports that RAD51 foci increase in cells that have helicase-dead $\operatorname{Pol} \theta(21,22)$. The robust RPA removal activity that we observed biochemically suggests that RPA clearance is a major target for Pol $\theta-h$ in cells.

The Poly-h domain is postulated to be a reverse helicase, or annealase, that can thermodynamically hybridize short microhomologies

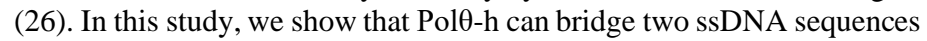
regardless of sequence homology. Based on this result, we suggest that the microhomology selection is mediated by the polymerase domain where Pol0-h initiates a 3' to 5' processive "microhomology scan" for the polymerase domain $(15,51)$. Surprisingly, these ssDNA bridges are highly resistant to $\mathrm{NaCl}$, suggesting that additional protein factors are required for their disassembly.

PARP-1 is one of the first DNA damage sensing proteins to localize to DNA damage (57). Pol $\theta$ recruitment to laser damage is reduced in cells with PARP inhibitors or PARP-1 depletion (22). Our data suggest that PARP-1 may further regulate the activity of Pol $\theta$ beyond recruitment. PARP-1 binds with high affinity to DSB and ss/dsDNA junctions $(58,59)$. We propose that the PARylation activity on Pol $\theta$ may aid in regulation and dissociation post-microhomology synthesis. Pol $\theta$ binds to the resected 3' ssDNA and processively translocases internally where PARP-1 then potentially PARylates Pol $\theta$. This may function in increasing the access to the polymerized DNA for ligation by the LIG3XRCC1 complex (60). Additionally, PARylation may aid in the iterative microhomology selection and multiple rounds of DNA synthesis via regulation of PolO DNA-binding $(15,61)$. We also do not rule out that PARylation by PARP-1 may inhibit Pol $\theta$ DNA binding to favor more accurate forms of repair. Together, this work shows that Pol $\theta$ plays multiple roles in mediating end-joining at DSBs in NHEJ/HR-deficient cancers and reiterates the importance of understanding the mechanistic functions of $\operatorname{Pol} \theta$ as a promising therapeutic target $(12,30-32)$.

\section{Materials and Methods}

Proteins and Nucleic Acids

Oligonucleotides were purchased from IDT or IBA (for fluorescent oligos). Polt-h (amino acids 1-894) was cloned into a pET19 vector with an N-terminal 6xHis-TwinStrep-SUMO tag to generate pIF378. Pol6-h(3A) mutations E121A, D216A, and E217A were cloned with primers IF733 and IF734 using QuikChange Lightning Multi SiteDirected Mutagenesis Kit to generate plasmid pIF585 (Agilent \#210516). RAD51(K133R) was mutagenized using inverse PCR with primers IF724 and IF725. RPA-GFP (plasmid pIF48), RAD51 (plasmid pIF224), RAD51(K133R) (plasmid pIF582) and PARP-1 (plasmid pIF662) were purified as previously described $(41,62,63)$.

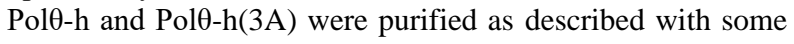
modifications (26). Plasmids were transformed into Rosetta(DE3) pLysS (Novagen) E. coli cells. Cell pellets were resuspended in Lysis Buffer (25 mM HEPES pH 8.0, $250 \mathrm{mM} \mathrm{NaCl}, 10 \mathrm{mM}$ imidazole pH 8.0, $5 \mathrm{mM}$ 2mercaptoethanol, $10 \%$ glycerol and supplemented with Roche cOmplete protease inhibitor) and sonicated. The lysed pellet was centrifuged at 40,000 rcf for 45 minutes. Resulting clarified lysate was placed on a HisTrap column (GE Healthcare) and eluted on a gradient from 10 - 250 $\mathrm{mM}$ imidazole. Eluted material was digested with SUMO Protease for two hours at $4^{\circ} \mathrm{C}$ and diluted with $25 \mathrm{mM}$ HEPES $\mathrm{pH} 8.0$ to a final $\mathrm{NaCl}$ concentration of $100 \mathrm{mM}$. This was passed through a heparin column (GE
Healthcare) and eluted with a gradient from $50-1000 \mathrm{mM} \mathrm{NaCl}$. Pure

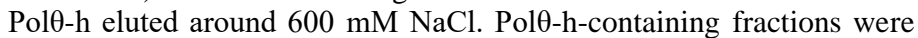
pooled, dialyzed in Dialysis Buffer (25 mM HEPES pH 8.0, $100 \mathrm{mM}$ $\mathrm{NaCl}, 5 \mathrm{mM}$ DTT, and $10 \%$ glycerol) for four hours at $4^{\circ} \mathrm{C}$. Pol $\theta-\mathrm{h}$ was spin concentrated and flash-frozen in liquid nitrogen.

\section{Single-molecule Microscopy}

Single-stranded DNA curtains were assembled in microfabricated flowcells according to published protocols $(35,36,64$, 65). All microscope experiments were conducted at $37^{\circ} \mathrm{C}$. Images were collected on an inverted Nikon Ti-E microscope in a prism TIRF configuration running NIS Elements (AR 4.30.02). Flowcells were illuminated with a 488 and $637 \mathrm{~nm}$ lasers (Coherent OBIS) split with a $638 \mathrm{~nm}$ dichroic mirror (Chroma). Two-color images were recorded by twin electron-multiplying charge coupled device (EMCCD) cameras (Andor iXon DU897). Uncompressed TIFF stacks were exported from NIS Elements and further analyzed in FIJI (66). Data analysis was performed in MatLab R2019a (MathWorks).

\section{RPA Removal Assays}

We first generated ssDNA in the flowcells as described previously $(35,65)$. Next, $0.4 \mathrm{nM}$ RPA-GFP was added to Imaging Buffer (40 mM Tris- $\mathrm{HCl}$ pH 8.0, $2 \mathrm{mM} \mathrm{MgCl} 2,1 \mathrm{mM}$ DTT, $0.2 \mathrm{mg} \mathrm{mL}$ ${ }^{1} \mathrm{BSA}, 50 \mathrm{mM} \mathrm{NaCl}$, and $1 \mathrm{mM} \mathrm{ATP}$ ) and injected at $0.4 \mathrm{~mL} \mathrm{~min}^{-1}$ to tether the ssDNA molecules at a chromium pedestal $13 \mu \mathrm{m}$ away from the biotinylated anchors. Unbound RPA-GFP washed out with Imaging Buffer. Pol $\theta$-h was introduced at the indicated concentration at a flow rate of $0.4 \mathrm{~mL} \mathrm{~min}{ }^{-1}$ and excess helicase flushed from the flowcell. To

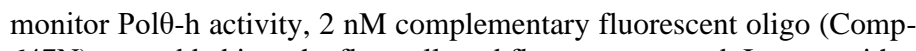
$647 \mathrm{~N}$ ) was added into the flowcell, and flow was stopped. Images with a $50 \mathrm{msec}$ exposure were acquired every 15 seconds using a $14 \mathrm{~mW} 488$ $\mathrm{nm}$ laser and $55 \mathrm{~mW} 637 \mathrm{~nm}$ laser (power measured at the front face of the prism). We fit the Atto647N fluorescent intensity along the DNA with a Gaussian function. The region of interest encompassed the ssDNA molecule and was three pixels wide to account for diffraction and ssDNA motion. The full-width at half-max (FWHM) of the Gaussian at each time point was used to measure the extent and rate of RPA-GFP clearance. For

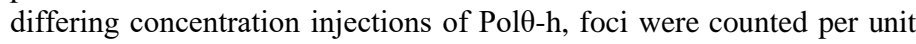
length of the ssDNA molecule. For experiments that quantified total fluorescence intensity, we measured this intensity along the length of the entire ssDNA and normalized to a unit length to correct for heterogeneity in the ssDNA lengths.

\section{RAD51 Removal Assays}

We first generated RPA-coated double-tethered ssDNA as described above. To assemble RAD51 filaments, $1 \mu \mathrm{M}$ RAD51(K133R) was injected in Imaging Buffer supplemented with $1 \mathrm{mM} \mathrm{CaCl}_{2}$ and flow was stopped for 10 minutes. Flow was resumed at $40 \mu \mathrm{L} \mathrm{min}^{-1}$ to remove unbound RAD51. Pol $\theta-\mathrm{h}$ was introduced at the indicated concentration and a flow rate of $0.4 \mathrm{~mL} \mathrm{~min}^{-1}$. Because of RAD51's strand capture activities, we could not use a fluorescent complementary oligo to monitor helicase translocation. Instead, we monitored RAD51(K133R) clearance by adding $2 \mathrm{nM}$ RPA-GFP to the flowcell. At this concentration, RPA cannot readily replace RAD51(K133R) on the ssDNA. Images with a 50 msec exposure are acquired every 15 seconds using a $40 \mathrm{~mW} 488 \mathrm{~nm}$ laser. We fit the GFP fluorescent intensity to a Gaussian distribution. The FWHM of the Gaussian distribution at each time point measured the extent and rate of RAD51(K133R) clearance. Fluorescent molecules were quantified as described for the RPA clearance experiments described above. 


\section{Polt-h Helicase Assays}

Pol $\theta$-h helicase activity was measured in flowcells containing double-stranded DNA (dsDNA), as used previously for RecQ-family helicases $(41,67)$. The DNA substrate was derived from bacteriophage $\lambda$. The $\cos L$ end was ligated with LAB07 and $\cos R$ with Lambda Poly-T

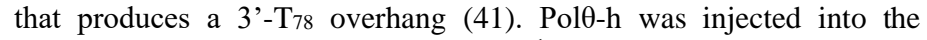
flowcell in Imaging Buffer at $0.4 \mathrm{~mL} \mathrm{~min}^{-1}$. Unbound Pol $\theta$-h was washed out and the buffer was switched to Imaging Buffer containing $0.1 \mathrm{nM}$ RPA-GFP at $0.4 \mathrm{~mL} \mathrm{~min}^{-1}$ to fluorescently label exposed ssDNA. The fluorescent intensity of RPA-GFP foci was calculated by averaging the area of a 3 x 3-pixel region of interest. We fluorescently stained DNA with YOYO-1 at the end of the experiment to confirm that RPA-GFP foci localized to DNA ends.

\section{DNA tethering assays}

For single-stranded capture experiments, we first generated ssDNA as described above. $0.4 \mathrm{nM}$ RPA-GFP is added to Imaging Buffer and flown through the flowcell at $0.4 \mathrm{~mL} \mathrm{~min}^{-1}$ to double-tether the ssDNA molecules. Unbound RPA-GFP was flushed out with Imaging

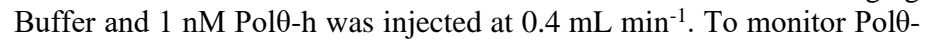
h oligo capture, $2 \mathrm{nM}$ noncomplementary fluorescent oligo (Noncomp$647 \mathrm{~N}$ ) was then added to the flowcell. Binding was monitored by acquiring 50 msec images every 15 seconds using $14 \mathrm{~mW} 488 \mathrm{~nm}$ laser and $55 \mathrm{~mW} 637 \mathrm{~nm}$ laser.

For double-stranded DNA end bridging experiments, we hybridized $\lambda$-phage DNA with LAB07 and Lambda Poly-T oligos by thermal melting and subsequent ligation with T4 DNA Ligase (NEB, M0202) as previously described (41). The DNA was fluorescently stained with YOYO-1 to visualize end-tethering.

For experiments involving PARP-1, $5 \mathrm{nM}$ Pol $\theta-\mathrm{h}$ was injected

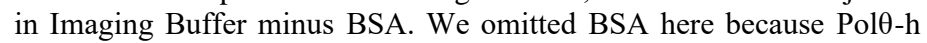
PARylation was inhibited in the presence of BSA in our single-molecule experiments, possibly because BSA can act as a competitor for PARP-1 activity. PARP-1 was labeled with an anti-HA primary and goat antimouse QDot705 secondary antibodies (ICL RHGT-45A-Z and Thermo Q-11461MP) and injected into the flowcell. Next, we switched to Imaging Buffer supplemented with $50 \mu \mathrm{M} \mathrm{NAD}^{+}$to initiate PARylation. End-tethering was monitored by acquiring $50 \mathrm{msec}$ images every 5 seconds using $14 \mathrm{~mW} 488 \mathrm{~nm}$ laser.

\section{Ensemble PARylation}

We performed Polt-h PARylation reactions in automodification buffer (30 mM HEPES pH 8.0, $50 \mathrm{mM} \mathrm{NaCl}, 1.5 \mathrm{mM}$ $\mathrm{MgCl}_{2}, 1 \mathrm{mM}$ DTT) with $1 \mu \mathrm{M}$ Pol $\theta$-h, $500 \mathrm{nM}$ PARP-1, 4.5 mM NAD ${ }^{+}$, $500 \mathrm{nM}$ annealed oligos (NJ061 and NJ062) at $30{ }^{\circ} \mathrm{C} \mathrm{(63).} \mathrm{Western} \mathrm{blots}$ were imaged on an Odyssey imaging system (Licor) with anti-PAR primary and goat anti-mouse IR680 (Millipore Sigma AM80 and Abcam ab216776, respectively). A dT 50 oligo was radioactively labeled with ${ }^{32} \mathrm{P}$ by T4 PNK (NEB M0201). EMSAs were performed in Imaging Buffer at room temperature. Pol $\theta-h$ ssDNA displacement EMSAs were performed in automodification buffer with 25 nM PARP-1.

\section{End Matter}

\section{Author Contributions and Notes}

J.M.S. and I.J.F. designed the experiments. J.M.S. performed the experiments and analyzed data. M.M.S. contributed to protein purification and data analysis. I.J.F. supervised the project. J.M.S., M.M.S., and I.J.F. wrote the manuscript.

\section{Acknowledgments}

We thank Drs. Richard Pomerantz, Marc Wold, and Mauro Modesti for sharing over-expression vectors, and to members of the Finkelstein lab for carefully reading this manuscript. Funding: This work is supported by CPRIT (RP190301 to I.J.F.) and the NIH (CA092584 to I.J.F.). Michael Soniat is supported by the American Cancer Society postdoctoral fellowship (PF-17-169-01-DMC). Competing interests: The authors declare no competing interests. Data and materials availability: All data in the manuscript or the supplementary material is available upon request.

\section{References}

1. Doherty,A.J. and Jackson,S.P. (2001) DNA repair: how Ku makes ends meet. Curr. Biol. CB, 11, R920-924.

2. Stinson,B.M. and Loparo,J.J. (2021) Repair of DNA Double-Strand Breaks by the Nonhomologous End Joining Pathway. Annu. Rev. Biochem., 10.1146/annurev-biochem-080320-110356.

3. Davis,A.J. and Chen,D.J. (2013) DNA double strand break repair via non-homologous end-joining. Transl. Cancer Res., 2, 130-143.

4. Graham,T.G.W., Walter,J.C. and Loparo,J.J. (2016) Two-Stage Synapsis of DNA Ends during Non-homologous End Joining. Mol. Cell, 61, 850-858.

5. Symington,L.S. and Gautier,J. (2011) Double-strand break end resection and repair pathway choice. Annu. Rev. Genet., 45, 247-271.

6. Sugiyama,T., Zaitseva,E.M. and Kowalczykowski,S.C. (1997) A Single-stranded DNA-binding Protein Is Needed for Efficient Presynaptic Complex Formation by the Saccharomyces cerevisiae Rad51 Protein. J. Biol. Chem., 272, 7940-7945.

7. McVey,M., Khodaverdian,V.Y., Meyer,D., Cerqueira,P.G. and Heyer,W.-D. (2016) Eukaryotic DNA Polymerases in Homologous Recombination. Annu. Rev. Genet., 50, 393-421.

8. Mathiasen,D.P. and Lisby,M. (2014) Cell cycle regulation of homologous recombination in Saccharomyces cerevisiae. FEMS Microbiol. Rev., 38, 172-184.

9. Kawale,A.S. and Sung,P. (2020) Mechanism and significance of chromosome damage repair by homologous recombination. Essays Biochem., 64, 779-790.

10. Schrempf,A., Slyskova,J. and Loizou,J.I. (2021) Targeting the DNA Repair Enzyme Polymerase $\theta$ in Cancer Therapy. Trends Cancer, 7, 98111.

11. Malaby,A.W., Martin,S.K., Wood,R.D. and Doublié,S. (2017) Chapter Five - Expression and Structural Analyses of Human DNA Polymerase $\theta$ (POLQ). In Eichman,B.F. (ed), Methods in Enzymology, DNA Repair Enzymes: Structure, Biophysics, and Mechanism. Academic Press, Vol. 592, pp. 103-121.

12. Roerink,S.F., Schendel,R. van and Tijsterman,M. (2014) Polymerase theta-mediated end joining of replication-associated DNA breaks in C. elegans. Genome Res., 24, 954-962.

13. Wood,R.D. and Doublié,S. (2016) DNA polymerase $\theta$ (POLQ), double-strand break repair, and cancer. DNA Repair, 44, 22-32.

14. Kent,T., Chandramouly,G., McDevitt,S.M., Ozdemir,A.Y. and Pomerantz,R.T. (2015) Mechanism of microhomology-mediated endjoining promoted by human DNA polymerase $\theta$. Nat. Struct. Mol. Biol., 22, 230-237.

15. Wyatt,D.W., Feng,W., Conlin,M.P., Yousefzadeh,M.J., Roberts,S.A., Mieczkowski,P., Wood,R.D., Gupta,G.P. and Ramsden,D.A. (2016) Essential Roles for Polymerase $\theta$-Mediated End Joining in the Repair of Chromosome Breaks. Mol. Cell, 63, 662-673.

16. He,P. and Yang,W. (2018) Template and primer requirements for DNA Pol $\theta$-mediated end joining. Proc. Natl. Acad. Sci. U. S. A., 10.1073/pnas.1807329115. 
Schaub et al. 2021 - preprint copy - BioRxiv

17. Truong,L.N., Li,Y., Shi,L.Z., Hwang,P.Y.-H., He,J., Wang,H., Razavian,N., Berns,M.W. and Wu,X. (2013) Microhomology-mediated End Joining and Homologous Recombination share the initial end resection step to repair DNA double-strand breaks in mammalian cells. Proc. Natl. Acad. Sci., 110, 7720-7725.

18. Zahn,K.E., Jensen,R.B., Wood,R.D. and Doublié,S. (2021) Human DNA polymerase $\theta$ harbors DNA end-trimming activity critical for DNA repair. Mol. Cell, 10.1016/j.molcel.2021.01.021.

19. Deng,S.K., Gibb,B., de Almeida,M.J., Greene,E.C. and Symington,L.S. (2014) RPA antagonizes microhomology-mediated repair of DNA double-strand breaks. Nat. Struct. Mol. Biol., 21, 405-412. 20. Jensen,R.B., Carreira,A. and Kowalczykowski,S.C. (2010) Purified human BRCA2 stimulates RAD51-mediated recombination. Nature, 467, 678-683.

21. Ceccaldi,R., Liu,J.C., Amunugama,R., Hajdu,I., Primack,B., Petalcorin,M.I.R., O'Connor,K.W., Konstantinopoulos,P.A., Elledge,S.J., Boulton,S.J., et al. (2015) Homologous-recombination-

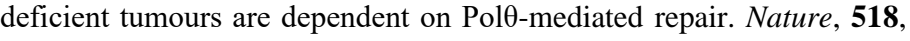
$258-262$.

22. Mateos-Gomez,P.A., Gong,F., Nair,N., Miller,K.M., LazzeriniDenchi,E. and Sfeir,A. (2015) Mammalian polymerase $\theta$ promotes alternative NHEJ and suppresses recombination. Nature, 518, 254-257. 23. Yousefzadeh,M.J. and Wood,R.D. (2013) DNA polymerase POLQ and cellular defense against DNA damage. DNA Repair, 12, 1-9.

24. Black,S.J., Kashkina,E., Kent,T. and Pomerantz,R.T. (2016) DNA Polymerase $\theta$ : A Unique Multifunctional End-Joining Machine. Genes, 7, 67.

25. Seki,M., Marini,F. and Wood,R.D. (2003) POLQ (Pol theta), a DNA polymerase and DNA-dependent ATPase in human cells. Nucleic Acids Res., 31, 6117-6126.

26. Mateos-Gomez,P.A., Kent,T., Deng,S.K., McDevitt,S., Kashkina,E., Hoang,T.M., Pomerantz,R.T. and Sfeir,A. (2017) The helicase domain of PolO counteracts RPA to promote alt-NHEJ. Nat. Struct. Mol. Biol., 24, 1116-1123.

27. Ozdemir,A.Y., Rusanov,T., Kent,T., Siddique,L.A. and Pomerantz,R.T. (2018) Polymerase $\theta$-helicase efficiently unwinds DNA and RNA-DNA hybrids. J. Biol. Chem., 293, 5259-5269.

28. Higgins,G.S., Harris,A.L., Prevo,R., Helleday,T., McKenna,W.G. and Buffa,F.M. (2010) Overexpression of POLQ confers a poor prognosis in early breast cancer patients. Oncotarget, 1, 175-184.

29. Kawamura,K., Bahar,R., Seimiya,M., Chiyo,M., Wada,A., Okada,S., Hatano,M., Tokuhisa,T., Kimura,H., Watanabe,S., et al. (2004) DNA polymerase theta is preferentially expressed in lymphoid tissues and upregulated in human cancers. Int. J. Cancer, 109, 9-16.

30. Lemée,F., Bergoglio,V., Fernandez-Vidal,A., Machado-Silva,A., Pillaire,M.-J., Bieth,A., Gentil,C., Baker,L., Martin,A.-L., Leduc,C., et al. (2010) DNA polymerase theta up-regulation is associated with poor survival in breast cancer, perturbs DNA replication, and promotes genetic instability. Proc. Natl. Acad. Sci. U. S. A., 107, 13390-13395.

31. Zhou,J., Gelot,C., Pantelidou,C., Li,A., Yücel,H., Davis,R.E., Farkkila,A., Kochupurakkal,B., Syed,A., Shapiro,G.I., et al. (2020) Polymerase Theta Inhibition Kills Homologous Recombination Deficient Tumors. bioRxiv, 10.1101/2020.05.23.111658.

32. Feng,W., Simpson,D.A., Carvajal-Garcia,J., Price,B.A., Kumar,R.J., Mose,L.E., Wood,R.D., Rashid,N., Purvis,J.E., Parker,J.S., et al. (2019) Genetic determinants of cellular addiction to DNA polymerase theta. Nat. Commun., 10, 4286.

33. Higgins,G.S., Prevo,R., Lee,Y.-F., Helleday,T., Muschel,R.J., Taylor,S., Yoshimura,M., Hickson,I.D., Bernhard,E.J. and McKenna,W.G. (2010) A small interfering RNA screen of genes involved in DNA repair identifies tumor-specific radiosensitization by POLQ knockdown. Cancer Res., 70, 2984-2993.
34. Newman,J.A., Cooper,C.D.O., Aitkenhead,H. and Gileadi,O. (2015) Structure of the Helicase Domain of DNA Polymerase Theta Reveals a Possible Role in the Microhomology-Mediated End-Joining Pathway. Struct. Lond. Engl. 1993, 23, 2319-2330.

35. Schaub,J.M., Zhang,H., Soniat,M.M. and Finkelstein,I.J. (2018) Assessing Protein Dynamics on Low-Complexity Single-Stranded DNA Curtains. Langmuir, 10.1021/acs.langmuir.8b01812.

36. Soniat,M.M., Myler,L.R., Schaub,J.M., Kim,Y., Gallardo,I.F. and Finkelstein,I.J. (2017) Next-Generation DNA Curtains for SingleMolecule Studies of Homologous Recombination. Methods Enzymol., 592, 259-281.

37. Brockman,C., Kim,S.J. and Schroeder,C.M. (2011) Direct observation of single flexible polymers using single stranded DNA. Soft Matter, 7, 8005-8012.

38. Lee,K.S., Marciel,A.B., Kozlov,A.G., Schroeder,C.M., Lohman,T.M. and Ha,T. (2014) Ultrafast Redistribution of E. coli SSB along Long Single-Stranded DNA via Intersegment Transfer. J. Mol. Biol., 426, 2413-2421.

39. Dillard,K.E., Schaub,J.M., Brown,M.W., Saifuddin,F.A., Xiao,Y., Hernandez,E., Dahlhauser,S.D., Anslyn,E.V., Ke,A. and Finkelstein,I.J. (2019) Sortase-mediated fluorescent labeling of CRISPR complexes. In Methods in Enzymology. Elsevier, Vol. 616, pp. 43-59.

40. Büttner,K., Nehring,S. and Hopfner,K.-P. (2007) Structural basis for DNA duplex separation by a superfamily-2 helicase. Nat. Struct. Mol. Biol., 14, 647-652.

41. Soniat,M.M., Myler,L.R., Kuo,H.-C., Paull,T.T. and Finkelstein,I.J. (2019) RPA Phosphorylation Inhibits DNA Resection. Mol. Cell, 75, 145-153.e5.

42. Ma,C.J., Gibb,B., Kwon,Y., Sung,P. and Greene,E.C. (2017) Protein dynamics of human RPA and RAD51 on ssDNA during assembly and disassembly of the RAD51 filament. Nucleic Acids Res., 45, 749-761.

43. Chi,P., Van Komen,S., Sehorn,M.G., Sigurdsson,S. and Sung,P. (2006) Roles of ATP binding and ATP hydrolysis in human Rad51 recombinase function. DNA Repair, 5, 381-391.

44. Sfeir,A. and Symington,L.S. (2015) Microhomology-Mediated End Joining: A Back-up Survival Mechanism or Dedicated Pathway? Trends Biochem. Sci., 40, 701-714.

45. Wang,M., Wu,W., Wu,W., Rosidi,B., Zhang,L., Wang,H. and Iliakis,G. (2006) PARP-1 and Ku compete for repair of DNA double strand breaks by distinct NHEJ pathways. Nucleic Acids Res., 34, 6170 6182.

46. Asher,G., Reinke,H., Altmeyer,M., Gutierrez-Arcelus,M., Hottiger,M.O. and Schibler,U. (2010) Poly(ADP-ribose) polymerase 1 participates in the phase entrainment of circadian clocks to feeding. Cell, 142, 943-953.

47. Lönn,P., van der Heide,L.P., Dahl,M., Hellman,U., Heldin,C.-H. and Moustakas,A. (2010) PARP-1 attenuates Smad-mediated transcription. Mol. Cell, 40, 521-532.

48. Luo,X., Ryu,K.W., Kim,D.-S., Nandu,T., Medina,C.J., Gupte,R., Gibson,B.A., Soccio,R.E., Yu,Y., Gupta,R.K., et al. (2017) PARP-1 Controls the Adipogenic Transcriptional Program by PARylating $\mathrm{C} / \mathrm{EBP} \beta$ and Modulating Its Transcriptional Activity. Mol. Cell, 65, 260271.

49. Wang,C., Zhang,F., Wang,L., Zhang,Y., Li,X., Huang,K., Du,M., Liu,F., Huang,S., Guan,Y., et al. (2013) Poly(ADP-ribose) polymerase 1 promotes oxidative-stress-induced liver cell death via suppressing farnesoid X receptor $\alpha$. Mol. Cell. Biol., 33, 4492-4503.

50. Hendriks,I.A., Larsen,S.C. and Nielsen,M.L. (2019) An Advanced Strategy for Comprehensive Profiling of ADP-ribosylation Sites Using Mass Spectrometry-based Proteomics. Mol. Cell. Proteomics MCP, 18, 1010-1026. 
bioRxiv preprint doi: https://doi.org/10.1101/2021.06.03.446937; this version posted June 3, 2021. The copyright holder for this preprint (which was not certified by peer review) is the author/funder. All rights reserved. No reuse allowed without permission.

Schaub et al. 2021 - preprint copy - BioRxiv

51. Carvajal-Garcia,J., Cho,J.-E., Carvajal-Garcia,P., Feng,W., Wood,R.D., Sekelsky,J., Gupta,G.P., Roberts,S.A. and Ramsden,D.A. (2020) Mechanistic basis for microhomology identification and genome scarring by polymerase theta. Proc. Natl. Acad. Sci. U. S. A., 117, 84768485 .

52. Koole,W., van Schendel,R., Karambelas,A.E., van Heteren,J.T., Okihara,K.L. and Tijsterman,M. (2014) A Polymerase Theta-dependent repair pathway suppresses extensive genomic instability at endogenous G4 DNA sites. Nat. Commun., 5, 3216.

53. Schimmel,J., Kool,H., van Schendel,R. and Tijsterman,M. (2017) Mutational signatures of non-homologous and polymerase thetamediated end-joining in embryonic stem cells. EMBO J., 36, 3634-3649. 54. Gibb,B., Ye,L.F., Gergoudis,S.C., Kwon,Y., Niu,H., Sung,P. and Greene,E.C. (2014) Concentration-Dependent Exchange of Replication Protein A on Single-Stranded DNA Revealed by Single-Molecule Imaging. PLoS ONE, 9, e87922-13.

55. Pokhrel,N., Caldwell,C.C., Corless,E.I., Tillison,E.A., Tibbs,J., Jocic,N., Tabei,S.M.A., Wold,M.S., Spies,M. and Antony,E. (2019) Dynamics and selective remodeling of the DNA-binding domains of RPA. Nat. Struct. Mol. Biol., 26, 129-136.

56. Nguyen,B., Sokoloski,J., Galletto,R., Elson,E.L., Wold,M.S. and Lohman,T.M. (2014) Diffusion of Human Replication Protein A along Single-Stranded DNA. J. Mol. Biol., 426, 3246-3261.

57. Yang,G., Liu,C., Chen,S.-H., Kassab,M.A., Hoff,J.D., Walter,N.G. and $\mathrm{Yu}, \mathrm{X}$. (2018) Super-resolution imaging identifies PARP1 and the Ku complex acting as DNA double-strand break sensors. Nucleic Acids Res., 46, 3446-3457.

58. D'Silva,I., Pelletier,J.D., Lagueux,J., D'Amours,D., Chaudhry,M.A., Weinfeld,M., Lees-Miller,S.P. and Poirier,G.G. (1999) Relative affinities of poly(ADP-ribose) polymerase and DNA-dependent protein kinase for DNA strand interruptions. Biochim. Biophys. Acta, 1430, 119-126.

59. Jorgensen,T.J., Chen,K., Chasovskikh,S., Roy,R., Dritschilo,A. and Uren,A. (2009) Binding kinetics and activity of human poly(ADP-ribose) polymerase-1 on oligo-deoxyribonucleotide substrates. J. Mol. Recognit. $J M R, 22,446-452$.

60. Dutta,A., Eckelmann,B., Adhikari,S., Ahmed,K.M., Sengupta,S., Pandey,A., Hegde,P.M., Tsai,M.-S., Tainer,J.A., Weinfeld,M., et al. (2017) Microhomology-mediated end joining is activated in irradiated human cells due to phosphorylation-dependent formation of the XRCC1 repair complex. Nucleic Acids Res., 45, 2585-2599.

61. Schimmel,J., van Schendel,R., den Dunnen,J.T. and Tijsterman,M. (2019) Templated Insertions: A Smoking Gun for Polymerase ThetaMediated End Joining. Trends Genet. TIG, 35, 632-644.

62. Brouwer,I., Moschetti,T., Candelli,A., Garcin,E.B., Modesti,M., Pellegrini,L., Wuite,G.J. and Peterman,E.J. (2018) Two distinct conformational states define the interaction of human RAD51-ATP with single-stranded DNA. EMBO J., 37.

63. Langelier,M.-F., Planck,J.L., Servent,K.M. and Pascal,J.M. (2011) Purification of human PARP-1 and PARP-1 domains from Escherichia coli for structural and biochemical analysis. Methods Mol. Biol. Clifton NJ, 780, 209-226.

64. Gallardo,I.F., Pasupathy,P., Brown,M., Manhart,C.M., Neikirk,D.P., Alani,E. and Finkelstein,I.J. (2015) High-Throughput Universal DNA Curtain Arrays for Single-Molecule Fluorescence Imaging. Langmuir, 31, 10310-10317.

65. Zhang,H., Schaub,J.M. and Finkelstein,I.J. (2020) RADX condenses single-stranded DNA to antagonize RAD51 loading. Nucleic Acids Res., 48, 7834-7843.

66. Schindelin,J., Arganda-Carreras,I., Frise,E., Kaynig,V., Longair,M., Pietzsch,T., Preibisch,S., Rueden,C., Saalfeld,S., Schmid,B., et al. (2012) Fiji: an open-source platform for biological-image analysis. Nat. Methods, 9, 676-682.

67. Rad,B., Forget,A.L., Baskin,R.J. and Kowalczykowski,S.C. (2015) Single-molecule visualization of RecQ helicase reveals DNA melting, nucleation, and assembly are required for processive DNA unwinding. Proc. Natl. Acad. Sci. U. S. A., 112, E6852-6861. 